Short reports

\title{
Acute respiratory distress secondary to Morgagni diaphragmatic herniation in an adult
}

\author{
Newton ACS Wong, Colin M Dayan, Jim Virjee, Kenneth W Heaton
}

\begin{abstract}
Summary
Diaphragmatic hernias of the Morgagni type are generally thought to be asymptomatic in adults. This traditional assumption led to a delay in diagnosing a Morgagni hernia as the cause of acute respiratory distress in a chronic schizophrenic man. While Morgagni hernias are usually considered to be long-standing, we present radiological evidence of an acutely expanding hernia. The patient's symptoms were relieved by surgical repair of the hernia. We advise caution before dismissing Morgagni hernias in adults as being long-standing and clinically insignificant.
\end{abstract}

Keywords: acute respiratory distress, Morgagni hernia, diaphragm

\section{Introduction}

Morgagni hernias are the least common of diaphragmatic hernias. ${ }^{1}$ In adults they are traditionally considered to be long-standing and clinically insignificant. There have been a few reports of such hernias presenting as bowel obstruction and/or with chest pain. ${ }^{1-4}$ However, it has never been shown that symptoms coincided with the acute development or expansion of a Morgagni hernia. In the following case, we were able to demonstrate progressive Morgagni-type herniation which, uniquely, resulted in acute respiratory distress in an adult.

Bristol Royal

Infirmary,

Marlborough Street,

Bristol BS2 8HW, UK

Department of

Medicine

NACS Wong

CM Dayan

KW Heaton

Department of

Radiology

J Virjee

Correspondence to

Dr KW Heaton, University of Bristol, Bristol Royal

Infirmary, Marlborough

Street, Bristol BS2 8HW,

UK

Accepted 30 August 1994

\section{Case report}

A 67-year-old chronic schizophrenic man presented in December 1993 with a four-day history of increasing dyspnoea and a dry cough. On admission, he was feverish, tachypnoeic and tachycardic, and had widespread expiratory wheezes and coarse crackles with reduced breath sounds over his right lower chest anteriorly. Chest radiographs revealed massive herniation of bowel into the right side of his chest through an anterior diaphragmatic defect but with some aerated lung tissue on that side (figure 1).

After discussion with the duty general surgical and thoracic surgical teams, we decided that the diaphragmatic hernia was probably long-standing and that the symptoms were caused chiefly by a chest infection. The patient was therefore treated with antibiotics and, initially, he improved.

The next day, we learnt that the patient had presented a week earlier to a neighbouring hospital with a suspected chest infection. The chest radiograph taken then was reported as showing bowel loops interposed between the liver and the diaphragm (Chiliaditis syndrome) with only slight basal collapse of the right lung (figure 2).

On the second day of the patient's admission, his condition deteriorated. He became increasingly breathless and previously normal arterial blood gases now showed profound hypoxia $\left(\mathrm{PO}_{2} 60 \mathrm{mmHg}, 8 \mathrm{kPa}\right)$. The patient's respiratory distress was attributed to expansion of his Morgagni hernia and an emergency laparotomy was performed.

Operation revealed transverse colon herniating via a Morgagni-type defect into the right chest with a completely collapsed right lung. No intraperitoneal adhesions were seen. The bowel was decompressed, the hernia reduced and the defect repaired.

The patient's right lung expanded fully within a day of surgery and, while postoperative recovery was complicated by wound infection and small bilateral pleural effusions, the patient was discharged symptom-free three weeks after surgery.

Three weeks after discharge, the patient developed diarrhoea; his stools were loose and greenish brown without frank blood. Over the next week, he developed abdominal distension and became lethargic and anorexic. By the end of this week, the patient was feverish and complained of abdominal pain. Peritonitis was diagnosed by the attending practitioner but the patient died before he could be transferred to a hospital. Autopsy revealed evidence of peritonitis with intraperitoneal pus and extensive adhesions. One large adhesion obstructed the proximal descending colon. Proximal to this, the colon was distended and there was a perforation at the splenic flexure. There was mucosal inflammation of the sigmoid colon and upper rectum. The diaphragmatic repair was intact and apart from some oedema, both lungs were normal. Histological examination showed no signs of ischaemia around the colonic perforation. The patient's death was attributed to peritonitis from a colonic perforation resulting from adhesion obstruction. 

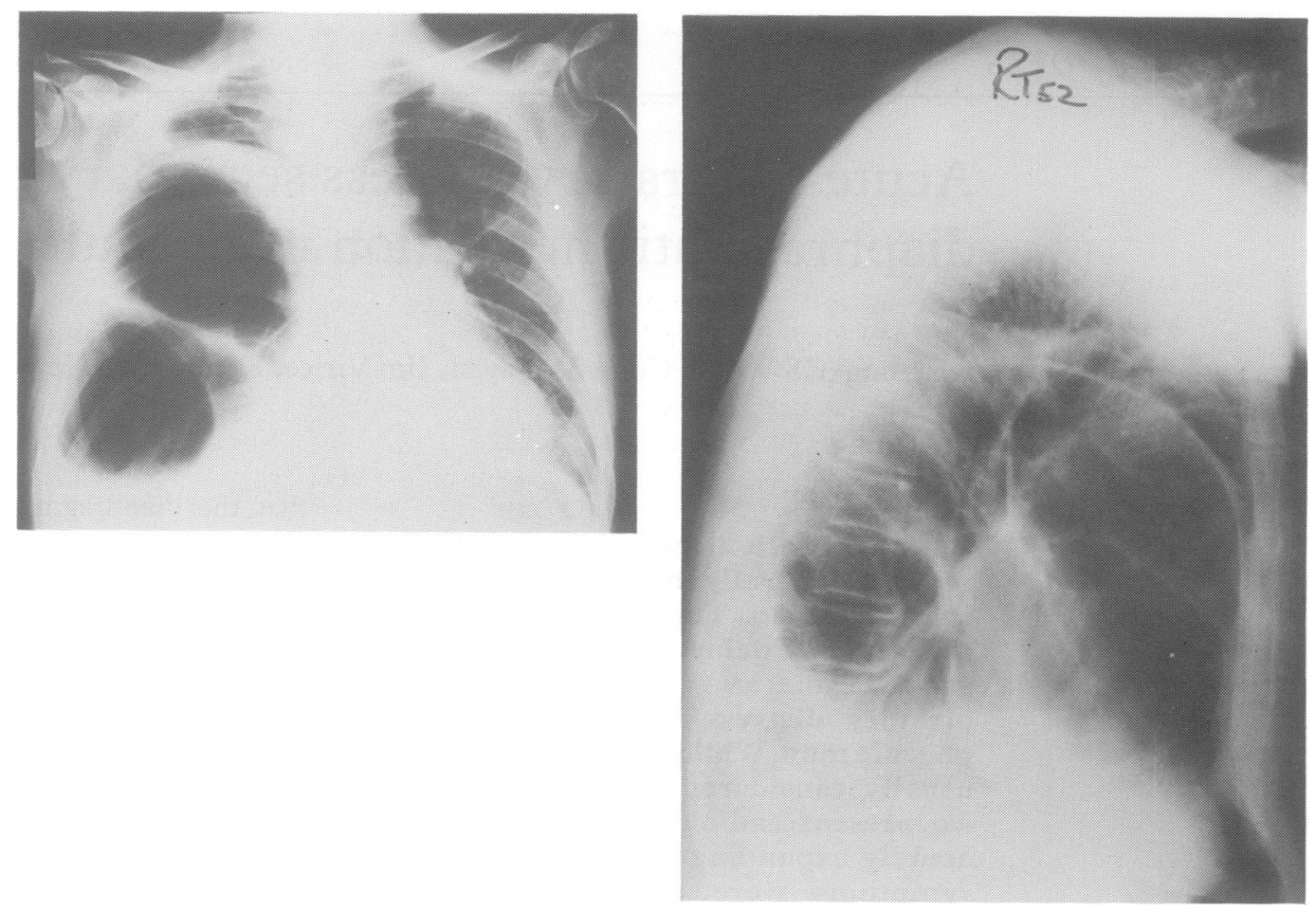

Figure 1 Chest radiographs taken on admission showing herniation of bowel into the right side of the chest (left) through an anterior diaphragmatic defect, as shown in the lateral film (right)

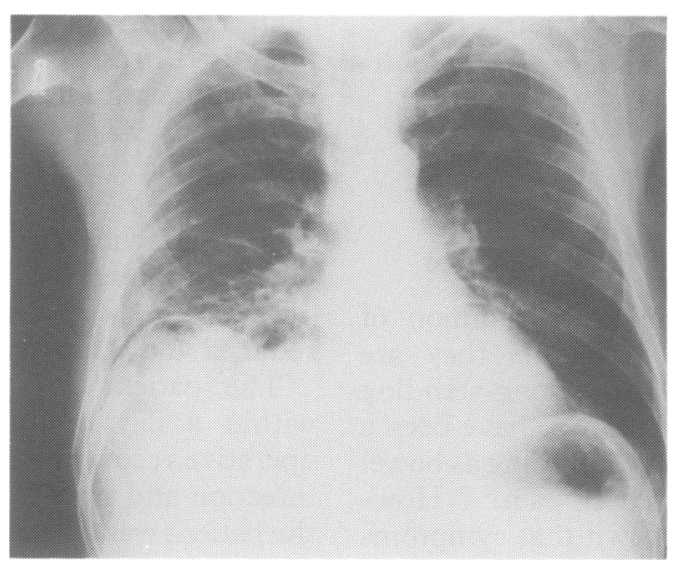

Figure 2 Chest radiograph taken one week prior to admission.

\section{Discussion}

To our knowledge, acute respiratory distress in an adult secondary to a Morgagni hernia has not been reported previously. Another special feature of this case is the radiological evidence of acute progression of the hernia.

The Morgagni diaphragmatic hernia was first described by Giovanni Morgagni in $1761 .^{1}$ Symptoms, if present, are usually abdominal pain, intestinal obstruction and/or chest tightness. ${ }^{1-4}$ However, the vast majority of the hernias are asymptomatic in adults and are only detected by radiological investigations for other purposes. ${ }^{2}$

These past observations led us to assume at first that our patient's Morgagni hernia was long-standing and not responsible for his dyspnoea. However, the chest radiograph taken a week prior to admission and the immediate expansion of the right lung postoperatively $\vec{\bullet}$ indicate that the collapse of this lung was of recent, as were his symptoms. His deterioration whilst in hospital may have been due to the lung collapsing completely from its partially inflated state on admission. There was no other pathology to explain the patient's sudden respiratory distress.

Schneidau and colleagues observed intermittent Morgagni-type herniation during a barium meal of a 29-year-old man complaining of episodic chest pain. ${ }^{4}$ However, this hernia was small and there was no statement that the patient was symptomatic each time the hernia was detected.

Although we have clear evidence of progression of our patient's hernia, it is less certain when the colon first herniated through the diaphragm. Indeed, the pre-admission chest radiograph may be interpreted as showing a small pre-existing hernia at the right lung base $N$ (figure 2). It is also not clear what precipitated the acute development and/or expansion of our patient's hernia. Past authors had proposed that sudden herniation may result from trauma, straining and heavy weightlifting.,5 As our patient was a poor communicator, we were not

\begin{tabular}{|l|}
\hline Morgagni diaphragmatic hernia \\
\hline - first described in 1761 \\
- defect between diaphragm's attachments to \\
- usually right-sided cartilages \\
- constitute $3 \%$ of diaphragmatic hernias \\
- usually asymptomatic
\end{tabular}


able to exclude such events. However, coughing induced by a respiratory tract infection may have increased his intra-abdominal pressure sufficiently to cause herniation. Any of the above events could also have caused further expansion of a pre-existing hernia. Such additional herniation may have been sufficient to produce outflow obstruction of the colon at the neck of the hernia, thus causing distension of the herniated colon and still further expansion of the hernia.

The relation of the patient's hernia to his final illness and death warrants further discussion. Strangulation of the herniated colon leading to ischaemia and thus perforation is unlikely for several reasons. First, symptoms of ischaemic bowel damage would have presented sooner. Second, the splenic flexure is unlikely to have been sufficiently mobile to enter a

1 Paris F, Tarazona V, Casillas M et al. Hernia of Morgagni. Thorax 1973; 28: 631-6.

2 Wolloch Y, Grunebaum M, Glanz I, Dintsman M. Symptomatic retrosternal (Morgagni) hernia. Am $₹$ Surg 1974; 127: $601-5$.

3 Valases C, Sills C. Case Report: Anterior diaphragmatic hernia (hernia of Morgagni). N₹ Med 1988; 7: 603-5. right-sided diaphragmatic hernia. Finally, there was no evidence of ischaemia around the perforation. The patient's diarrhoea may have resulted from his distal colitis, whose aetiology is unknown but could have been related to oral antibiotics given five weeks previously. However, the colitis is unlikely to have contributed to the patient's demise nor to have been directly linked to his Morgagni hernia. We suggest that the hernia was related to the perforation only because the surgical repair led to intraperitoneal adhesions.

In summary, we report the case of an acutely expanding Morgagni hernia precipitating respiratory distress in an adult. Our experience leads us to advise caution before dismissing Morgagni hernias in adults as being longstanding and clinically insignificant.

4 Schneidau A, Baron HJ, Rosin RD. Morgagni revisited: a case of intermittent chest pain. $\mathrm{Br} \mathcal{F}$ Radiol 1982; 55: 238-40.

5 Ellyson JH, Parks SN. Hernia of Morgagni in a trauma patient. $\mathcal{F}$ Trauma 1986; 26: 569-70. 